\title{
Molecular characterization of the uncultivatable hemotropic bacterium Mycoplasma haemofelis
}

\author{
Emily N Barker ${ }^{1 *}{ }^{*}$, Alistair C Darby ${ }^{2+}$, Chris R Helps ${ }^{1}$, lain R Peters ${ }^{1}$, Kate J Heesom ${ }^{3}$, Christopher J Arthur ${ }^{4}$, \\ Ben Crossett ${ }^{5}$, Margaret A Hughes ${ }^{2}$, Alan D Radford ${ }^{6}$ and Séverine Tasker $^{1}$
}

\begin{abstract}
Mycoplasma haemofelis is a pathogenic feline hemoplasma. Despite its importance, little is known about its metabolic pathways or mechanism of pathogenicity due to it being uncultivatable. The recently sequenced M. haemofelis str. Langford 1 genome was analysed and compared to those of other available hemoplasma genomes.

Analysis showed that in hemoplasmas genes involved in carbohydrate metabolism are limited to enzymes of the glycolytic pathway, with glucose appearing to be the sole energy source. The majority of the pentose phosphate pathway enzymes that catalyze the de novo synthesis of ribonucleotides were absent, as were cell division protein FtsZ and chaperonins GroEL/ES. Uncharacterized protein paralogs containing putative surface expression motifs, comprised $62 \%$ of M. haemofelis and $19 \%$ of Mycoplasma suis genome coverage respectively, the majority of which were present in a small number of unstructured islands. Limited mass spectrometry and immunoblot data matched a number of characterized proteins and uncharacterized paralogs, confirming their expression and immunogenicity in vivo.

These data have allowed further characterization of these important pathogens, including their limited metabolic capabilities, which may contribute to their uncultivatable status. A number of immunogenic proteins, and a potential mechanism for host immune system evasion, have been identified.
\end{abstract}

\section{Introduction}

The hemotropic mycoplasmas (hemoplasmas) are a group of bacteria that can induce hemolytic anemia in a wide variety of mammals [1]. The feline hemoplasma, $M y c o-$ plasma haemofelis, and the porcine hemoplasma, Mycoplasma suis were reclassified as members of the genus Mycoplasma within the Mollicutes class following 16S ribosomal RNA gene phylogenetic analysis [1]. Recently the whole genome sequence of $M$. haemofelis str. Langford 1 was published [2]. This low-passage strain has been shown to induce hemolytic anemia in immunocompetent specific pathogen free (SPF)-derived cats $[3,4]$. Subsequently the annotated genome sequences of two strains of $M$. suis and a further strain of $M$. haemofelis have also been published [5,6].

To date the hemoplasmas have been uncultivatable in vitro, meaning that sourcing large quantities of purified

\footnotetext{
* Correspondence: emi.barker@bristol.ac.uk

+ Contributed equally

${ }^{1}$ School of Veterinary Sciences, University of Bristol, Langford, BS40 5DU, UK

Full list of author information is available at the end of the article
}

hemoplasma DNA has been difficult. When hemoplasma shotgun libraries have been screened and compared against mass spectrometry data the lack of whole genome sequence data available for hemoplasmas, and the extensive host protein contamination present in preparations, have limited identification of hemoplasma proteins $[7,8]$.

The aim of this study was to fully describe the genome of M. haemofelis str. Langford 1, and to compare it with available hemoplasma genomes.

\section{Materials and methods}

Sources of $M$. haemofelis DNA and protein

Preparations of $M$. haemofelis str. Langford 1 had been previously purified from blood taken from an experimentally infected SPF-derived cat at a time of high parasitemia [9]. DNA and proteins were purified as previously described [7].

\section{Genome sequencing, analysis and annotation}

Whole shotgun pyrosequencing, genome closure and annotation was performed as described elsewhere [2].

\section{Biomed Central}


The genome was submitted to the European Molecular Biology Laboratory (EMBL) nucleotide sequence database (accession number FR773153).

\section{Genome sequence data for comparisons}

The genome sequences of $M$. suis str. KI_3806 (FQ790233), M. suis str. Illinois (CP002525), and M. haemofelis Ohio2 (CP002828) were obtained from GenBank. Artemis v12 was used to organize data and facilitate annotation [10]. Orthologs and paralogs were defined using OrthoMCL [11]. Repeat identification was made using MUMmer [12]. Homology was defined as $\mathrm{E} \leq 10^{-5}$.

\section{Identification of $M$. haemofelis str. Langford 1 proteins}

Mycoplasma haemofelis str. Langford 1 proteins were prepared for two-dimensional (2D) sodium dodecyl sulfate polyacrylamide gel electrophoresis (SDS-PAGE) using the 2D-clean up kit (GE Healthcare, Amersham Place, Little Chalfont, UK). Proteins were separated according to their isoelectric points on Immobiline ${ }^{\mathrm{TM}}$ DryStrip pH 3-11 NL (GE Healthcare) and then according to mass (Criterion XT 4-12\% Bis-Tris Precast Gels; Bio-Rad Laboratories Pty. Ltd., Gladesville, NSW Australia) alongside a molecular weight marker (Unstained Precision Plus Protein ${ }^{\mathrm{TM}}$ Standards, Bio-Rad Laboratories Ltd., Hemel Hempstead, UK). Gels were stained using Sypro ${ }^{\circledR}$ Ruby Protein Gel Stain (Invitrogen, Paisley, UK). Selected protein spots were picked for trypsin-digestion and the resulting peptides analyzed by liquid chromatography-tandem mass spectrometry (LC-MSMS; QSTAR Elite Q-TOF; AB Sciex Australia Pty. Ltd., Mt Waverley, Victoria, Australia). In addition, data derived from an earlier study using matrix assisted laser desorption/ionization tandem mass spectrometry (MALDI-MSMS) of immunoreactive protein spots were available [7]. Mass spectrometry (MS) data were analyzed using Mascot (Matrix Science Ltd, London, UK) to search a database of in silico predicted M. haemofelis proteins, using the following parameters: error tolerance of $0.2 \mathrm{Da}$ for precursor and product ion masses; 1 missed tryptic cleavage; allowing oxidation of methionine and propionamide modification of cysteine as optional modifications. Confident matches were defined by the Mascot score and statistical significance $(p<0.05)$.

\section{Results}

The general features of the M. haemofelis str. Langford 1 genome are shown in Figure 1, and these features are compared to other available hemoplasma genomes $(M$. haemofelis str. Ohio2, M. suis str. Illinois \&M. suis str. KI_3806) in Table 1. Both hemoplasmas contained single copies of the 16S, $23 \mathrm{~S}$ and $5 \mathrm{~S}$ ribosomal RNA (rRNA) genes, which were located in a single rRNA operon in $M$. haemofelis but not M. suis. A single copy of the ribonuclease $\mathrm{P} \beta$-subunit ribosomal gene was located in each hemoplasma upstream of, and on the complementary strand to, the ATP dependent protease La gene (lon). Transfer RNA (tRNA) genes corresponding to each of the amino acids were present in both species, including one assigned to the UGA (opal) codon, consistent with a tryptophan insertion at this position (M. haemofelis str. Langford 1 codon usage data: Additional file 1 Table S1). Both $M$. suis strains contained a lysine tRNA complementary to the AAG codon which was absent in M. haemofelis, whilst lysine tRNA complementary to the AAA codon was present in both species. From transitions in GC skew, and gene distribution we inferred that the $M$. haemofelis origin of replication is upstream of $d n a A$ (HF1_00010) as was also seen for $M$. suis. Using the mycoplasma genetic code Table 1545 putative open reading frames (ORFs) were identified in the $M$. haemofelis str. Langford 1 with a mean length of 743 nucleotides, of which 328 (21.2\% total ORFs) were homologs of proteins from non-hemoplasma bacterial species.

Of the pentose phosphate pathway enzymes required for de novo ribonucleotide synthesis, only the gene encoding ribose phosphate pyrophosphokinase (prs) was present in the hemoplasmas; genes encoding transketolase, ribose-5phosphate isomerase and ribulose-phosphate 3-epimerase were not identified. Alternative purine synthesis pathway homologs inosine-5 monophosphate dehydrogenase (GuaB) and guanosine 3, 5 monophosphate synthetase (GuaA) were however found in both $M$. haemofelis and M. suis, whilst homologs for pyrimidine synthesis enzymes cytidylate kinase (cmk) and adenosine kinase (HF1_14770) were only found in $M$. haemofelis.

The phosphotransferase system of Mollicutes appears to be present in hemoplasmas, including genes encoding glucose-specific components ( $p t s G \& c r r$ ) and the regulatory protein kinase (hprK). Phosphocarrier protein $\mathrm{HPr}(p t s H)$ of both species contain the His-15 site of enzyme I phosphorylation and the Ser-46 site for HprK phosphorylation. Genes encoding enzymes of the Embden-Meyerhof-Parnas glycolytic pathway catalyzing the conversion of glucose into pyruvate/lactate (Additional file 2 Figure S1) were present in both species whilst genes of the pyruvate dehydrogenase complex were absent, as were genes encoding enzymes of the citric acid cycle. Genes encoding the highly conserved $\mathrm{F}_{\mathrm{O}} \mathrm{F}_{1}$-ATP synthase complex (atpABCDEFGH) were present to complete the energy pathway, and maintain the transmembrane proton gradient in both species.

Transporters and pathways for the utilization of other energy sources appear to be partial or absent: the glycerol kinase gene $(g l p K)$ is present whilst the glycerol facilitator gene $(g l p F)$ is not. Genes required for fructose and mannitol uptake and metabolism (e.g. 1-phosphofructokinase; mannitol-1-phosphate 5-dehydrogenase) were not identified.

Minimal lipid transport and metabolism enzymes were identified. Those that were identified in all three 


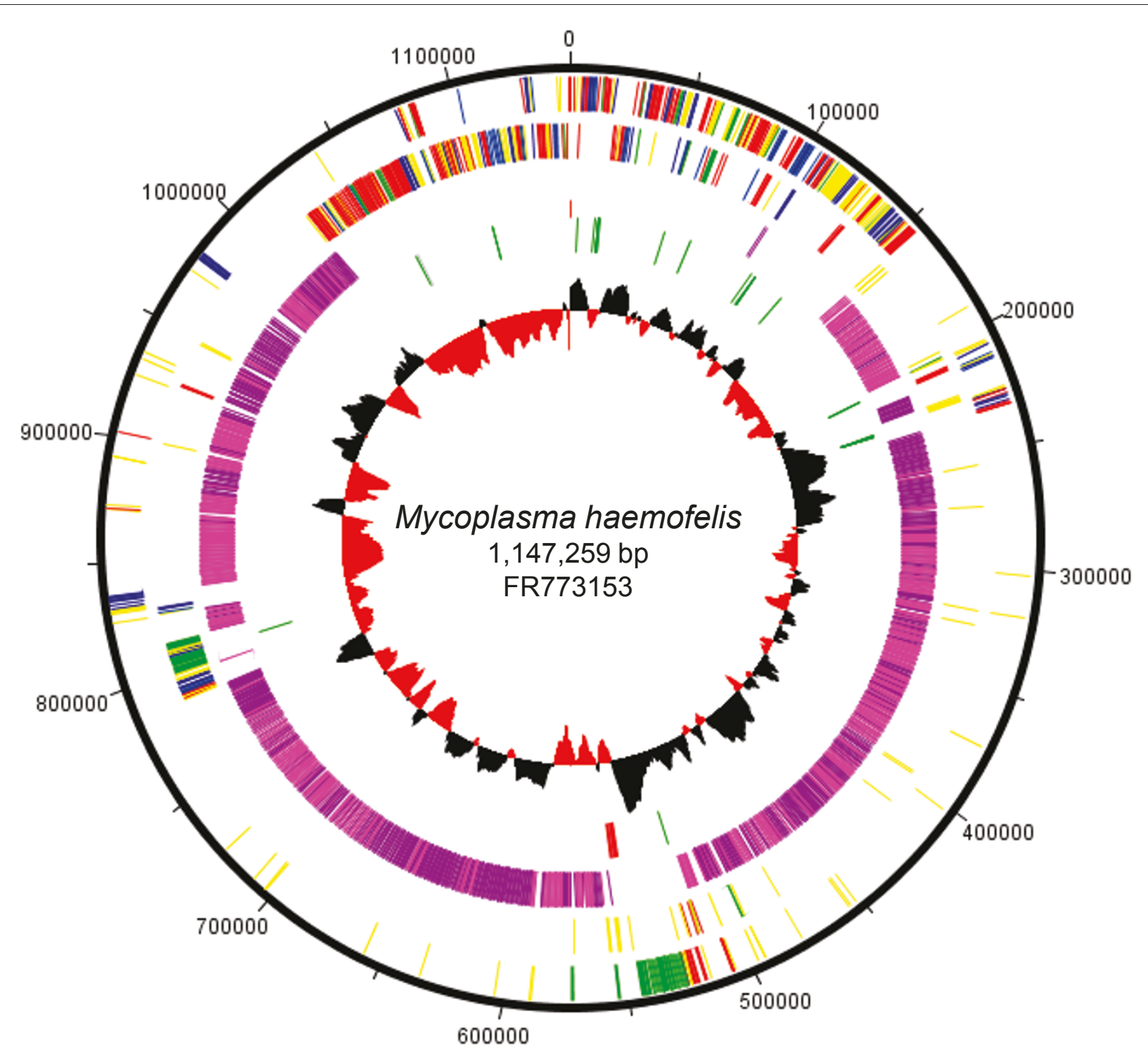

Figure 1 Circular representation of the Mycoplasma haemofelis str. Langford 1 genome. Outer concentric circle: genomic positions in bases, where position one is the first base of the $\boldsymbol{d n a A}$ gene. Second concentric circle: the predicted genes on the positive strand (excluding uncharacterized paralog genes). Third concentric circle: the predicted genes on the negative strand (excluding uncharacterized paralog genes). Information storage and processing (red), cellular processes and signaling (green), metabolism (blue), and poorly characterized (yellow). Fourth concentric circle: uncharacterized paralog genes (pink on the forward strand, purple on the negative strand). Fifth concentric circle: tRNA (green), rRNAs (red) and ribonuclease P ribosomal subunit (blue). Sixth concentric circle: the GC-skew diagram; where the black color indicates that the leading strand contains more Gs than Cs, and the red color indicates the more Cs than Gs.

genomes included cardiolipin synthase $(c l s)$ and choline kinase (licA), whilst phosphatidylglycerophosphate synthase ( $p g s A)$ was truncated in M. suis str. KI_3806.

Genome data also suggests that hemoplasmas have minimal amino acid synthesis capabilities, and that they are unable to de novo synthesize folate. In contrast, the highly conserved genes encoding the polyamine (spermidine/putrescine) $\mathrm{ABC}$ transporter complex (pot $A B C D E$ ) were identified in both species, as were coding sequences necessary for the synthesis of all aminoacyl-tRNAs, except glutaminyl-tRNA. In the absence of glutaminyltRNA synthetase, genes encoding the aspartyl-tRNA/glutamyl-tRNA amidotransferase ( gat $A B)$ to convert glutamyl-tRNA into glutaminyl-tRNA were identified in both species.

The $\mathrm{ABC}$ phosphate transport system permease genes (pst $A C \& B$ ) and other $\mathrm{ABC}$ transporter components were also identified including a ferrichrome $A B C$ 
Table 1 General attributes of the sequenced hemoplasma genomes

\begin{tabular}{|c|c|c|c|c|}
\hline Hemoplasma species & $\begin{array}{l}\text { Mycoplasma } \\
\text { haemofelis } \\
\text { str. Langford } 1\end{array}$ & $\begin{array}{l}\text { Mycoplasma } \\
\text { haemofelis } \\
\text { str. Ohio2 }\end{array}$ & $\begin{array}{l}\text { Mycoplasma suis } \\
\text { str. KI_3806 }\end{array}$ & $\begin{array}{l}\text { Mycoplasma } \\
\text { suis } \\
\text { str. Illinois }\end{array}$ \\
\hline EBML accession number & FR773153 & CP002828 & FQ790233 & CP002525 \\
\hline Topology (chromosome) & Circular & circular & circular & circular \\
\hline Length (nucleotides) & 1147259 & 1155937 & 709270 & 742431 \\
\hline $\mathrm{G}+\mathrm{C}$ content $(\%)$ & 38.9 & 38.8 & 31.1 & 31.1 \\
\hline $1^{\text {st }}$ codon & 43.5 & 43.5 & 41.0 & 41.1 \\
\hline $2^{\text {nd }}$ codon & 37.6 & 37.6 & 33.1 & 33.3 \\
\hline $3^{\text {rd }}$ codon & 36.0 & 35.9 & 20.3 & 20.1 \\
\hline Genes per kb & 1.35 & 1.24 & 1.12 & 1.14 \\
\hline $\begin{array}{l}\text { Mean coding density \% (mean gene density \% i.e. including } \\
\text { ribosomal genes) }\end{array}$ & $94.9(95.8)$ & $94.3(95.0)$ & $87.3(90.1)$ & $89.1(90.1)$ \\
\hline Putative ORFs & 1545 & $\begin{array}{l}1548 \text { (inc. } 25 \\
\text { pseudo) }\end{array}$ & $\begin{array}{l}794 \text { (inc. } 15 \\
\text { pseudo) }\end{array}$ & $\begin{array}{l}844 \text { (inc. } 5 \\
\text { pseudo) }\end{array}$ \\
\hline Uncharacterized ORFs (of which were identified as paralogs) & $1228(1115)$ & $1251(1,013)$ & $523(229)$ & $550(240)$ \\
\hline Structural RNAs & 35 & 35 & 36 & 36 \\
\hline Ribosomal RNA (16S; 5 S and 23S) & 3 & 3 & 3 & 3 \\
\hline Transfer RNA & 31 & 31 & 32 & 32 \\
\hline Ribonuclease $\mathrm{P} \beta$-subunit gene & 1 & 1 & 1 & 1 \\
\hline
\end{tabular}

ORF = open reading frame

transporter complex. Predicted proteins with homology to cation transporter proteins including those for cobalt $\left(\right.$ cbiO $\left._{1} \mathrm{O}_{2} Q\right)$, magnesium $(m g t E)$, potassium $(\operatorname{trk} A B)$ and sodium/calcium were also found.

A gene encoding a DHH family phosphoesterase homologous to $m g p A$ was present in both hemoplasma species; however no genes encoding other terminal organelle proteins of the pneumoniae group of mycoplasmas were identified. Homologs to M. suis proteins HspA1, MSG1 and enolase were predicted in $M$. haemofelis as chaperone protein DnaK, NAD-dependent glyceraldehyde-3-phosphate dehydrogenase and enolase respectively. Genes encoding the components of the chaperonin complex GroEL/ES were not identified in either hemoplasma species, nor was cell-division protein FtsZ.

No predicted $M$. haemofelis ORFs had significant matches to proteins known to specifically mediate colonisation, motility, chemotaxis, natural competence, antibiotic resistance or toxicity in other bacteria, including the P97like protein of $M$. suis which has a region homologous to Mycoplasma hyopneumoniae adhesin P97. In addition to cytidylate kinase and adenosine kinase, the $M$. haemofelis genome also contained genes encoding $\mathrm{Mn} / \mathrm{Fe}$ - superoxide dismutase $(\operatorname{sod} A)$, and tRNA modification GTPase (mnmEg), which were absent from both $M$. suis strains.

Of the 1228 (79.5\% of total ORFs) uncharacterized hypothetical proteins, 1115 (72.2\% of total ORFs), covering $61.9 \%$ of the $M$. haemofelis str. Langford 1 genome, were highly repeated protein paralogs (Additional file 3 Table S2) mostly contained within three large islands
(Figure 1: pink/purple bars). These protein paralogs could be seen as clusters of spots in the in silico predicted proteome of $M$. haemofelis str. Langford 1 (Figure 2a) and M. haemofelis str. Ohio2 (data not shown). Similar protein paralogs were detected in both M. suis str. KI_3806 (Figure 2b) and M. suis str. Illinois (data not shown) and in silico predicted proteomes, which contained 550 and 523 uncharacterized hypothetical proteins (including 240 and 229 paralogs; representing $18.9 \%$ and $18.5 \%$ genome coverage) respectively. Sequence comparisons suggested that they may have arisen by gene duplication events. The predicted motifs of the majority of these putative proteins were consistent with them being expressed on the cell surface; an $\mathrm{N}$-terminal signal peptide or transmembrane region followed by a non-cytoplasmic tail of approximately 200 amino acids. These paralogs were arranged islands flanked by homologs of prolipoproteins, ATP-dependent DNA helicase UvrD/PcrA, C-5 cytosine-specific DNA methylase or genes of the type I restriction-modification system (see Additional file 4 Figure S2 for example of protein paralog "island"). Differences in gene number and genome size between the different strains of hemoplasma species were almost entirely accounted for by differences in the number of genes encoding uncharacterized proteins, mostly those belonging to paralog groups. All characterized genes present in M. haemofelis str. Langford 1 were present in $M$. haemofelis str. Ohio 2 , and visa versa. Only one characterized gene, type II restriction enzyme DpnII, was present in $M$. suis str. 

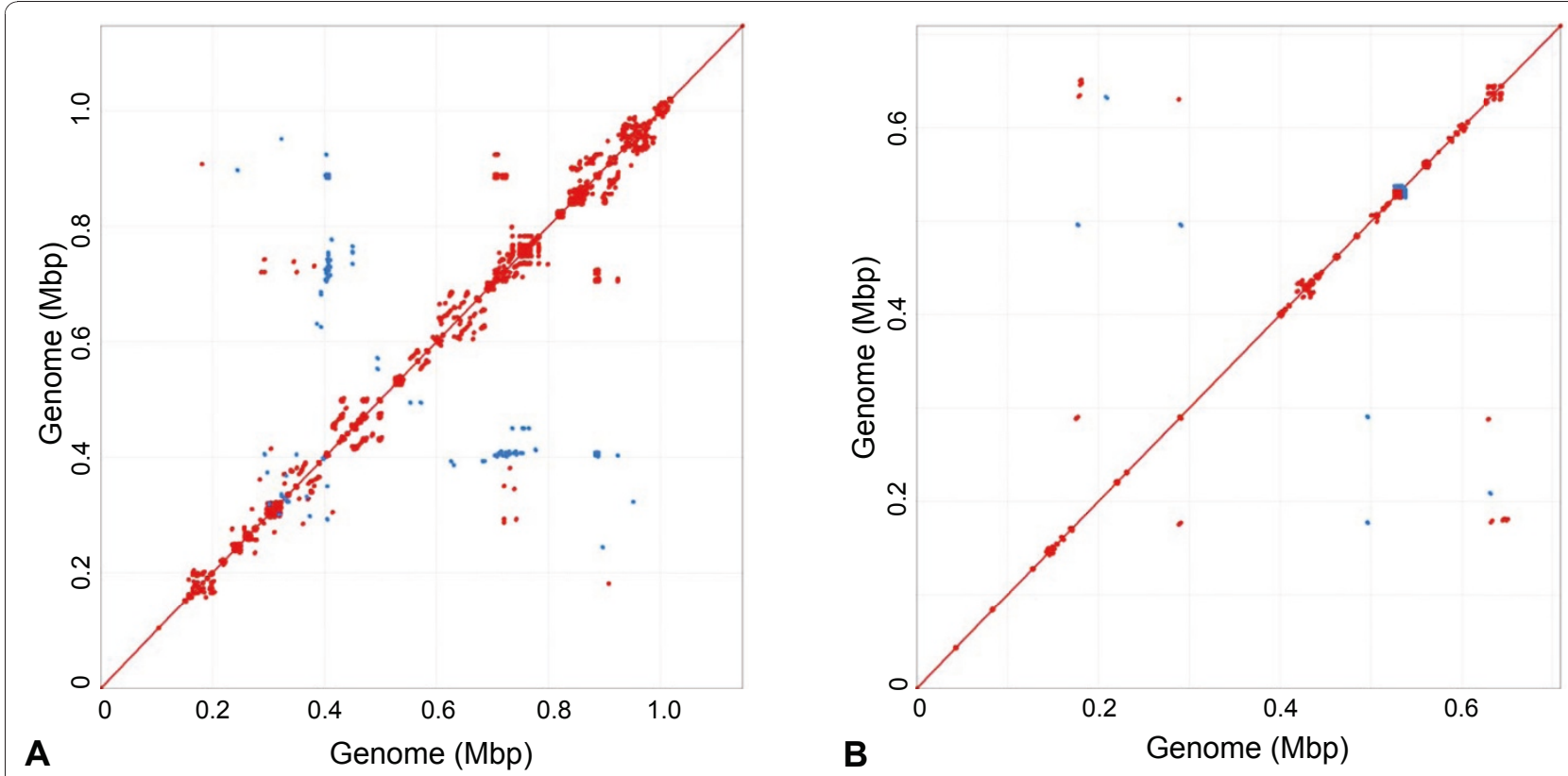

Figure 2 PROmer dotplot matrix of intra-genomic protein comparisons of Mycoplasma haemofelis str. Langford 1 (A) and Mycoplasma suis. str. KI_3806 (B). The genomes were BLASTx compared against themselves. Each dot represents a significant protein match: forward matches are colored red and reverse matches colored blue. Dots present on either side of the diagonal line represent repeated proteins.

Illinois but not $M$. suis str. KI_3806. Whole genome comparison between strains of $M$. haemofelis identified a $17.4 \mathrm{~kb}$ inversion event in a region of the genome containing genes encoding uncharacterized paralogs and other uncharacterized proteins. No large inversion events were detected between strains of M. suis.

Nineteen proteins were identified by comparing MS data of selected proteins to in silico predicted ORFs (Additional file 5 Table S3). Of these, seven were matched to characterized proteins, two to conserved hypothetical proteins and the remainder to uncharacterized hypothetical proteins. Of the hypothetical protein matches the strongest (Mascot score of 249; where > 14 was significant) was to hypothetical protein CBY92783, itself located within a string of repeated proteins, contiguous with its sole paralog (CBY92784). Six of the remaining hypothetical protein matches were to members of different protein paralog groups, one of which was known to correspond to an immunogenic protein spot.

\section{Discussion}

The recent publication of the first whole hemoplasma genome sequences $[2,5,6]$ represents a significant step forward in the study of these pathogens. Noticeable differences between the two hemoplasma species include the genome size, $1.15 \mathrm{Mbp}$ for $M$. haemofelis c.f. 0.71-0.74 Mbp for M. suis, and $\mathrm{G}+\mathrm{C}$ content, $38.8-38.9 \%$ for $M$. haemofelis $\mathrm{c}$. f. $31.1 \%$ for $M$. suis. The differences in genome length can mostly be accounted for by variation in the numbers of the hypothetical proteins, $1228-1251$ in M. haemofelis c.f. 523-550 in M. suis, whilst variation at the third codon position of almost $16 \%$, accounts for the majority of the $\mathrm{G}+\mathrm{C} \%$ difference.

Mycoplasma haemofelis was also found to have a very low percentage of intergenic regions with ORFs covering 94.9-94.3\% of the genome, and ribosomal genes a further $0.9 \%$. In contrast, the mean coding density of M. suis strains were $87.3-89.1 \%$, whilst pneumoniae group mycoplasmas, to which the hemoplasmas are most closely related based on ribosomal gene phylogeny [13], have mean genome coding densities ranging from $88.3 \%$ for Mycoplasma gallisepticum (AE015450) to $91.0 \%$ for Mycoplasma genitalium (L43967).

Mollicutes have previously been reported as bacteria having genomes comprising some of the highest proportions of protein paralogs [14]; for example $22.7 \%$ of the genome of plant pathogen "Candidatus Phytoplasma asteris" strain OY-M [15] and 15.3\% of the genome of porcine respiratory pathogen $M$. hyopneumoniae [16] encode protein paralogs. In comparison, $M$. haemofelis has a dramatically high number of hypothetical protein paralogs representing $61.9-62.7 \%$ genome coverage, whilst $M$. suis had only $18.5-18.9 \%$ genome coverage by hypothetical protein paralogs. Like in M. suis [6] the paralogs of $M$. haemofelis were present mostly within a small number of islands or regions within the genome which did not contain housekeeping genes. 
Hemoplasmas experience different environments during the infection cycle: surviving in host tissues and during presumed arthropod vector transmission $[17,18]$. The small number of sigma factors and transcription regulators identified would suggest a minimal ability to cope with survival outside of the mammalian host, which could be consistent with arthropods only providing a mechanical vector of transmission. The apparent reliance on glucose as the sole carbohydrate energy source would also be consistent with the hemoplasmas being highly adapted to the fairly constant epierythrocytic environment. All cultivatable mycoplasmas for which genome sequence data is available have an intact pentose phosphate pathway for the de novo synthesis of ribonucletoides, whilst the uncultivatable phytoplasmas do not and rely on host cells as a source of ribonucleotides. The absence of an intact pentose phosphate pathway could contribute to the uncultivatable status of the hemoplasmas. However, the presence of genes in M. haemofelis, and to a lesser extent in $M$. suis, encoding enzymes that could form alternative synthesis pathways indicate that ribonucleotide metabolism in hemoplasmas requires further investigation, and may not be the same for both species.

The transporter proteins identified indicate that, in addition to glucose, hemoplasmas have the potential to source amino acids, glucose, phosphates and cations from their environment. Mechanisms for the uptake of bases or nucleotides were not identified; however these could be present in the $\mathrm{ABC}$ transporters for which substrates were not indentified or in the hypothetical proteins. This finding is similar to other Mollicutes, which are also presumed to source nucleotides from their environment.

Homologs of $\operatorname{sodA}$, rnr and $m g p A$ genes had previously been identified during preliminary screening of the $M$. haemofelis genome as potential virulence factors [19]. Superoxide dismutase activity could represent an important antioxidant defense of $M$. haemofelis to the high oxygen tension in the epierythrocytic environment through catalysis of dismutation of superoxide into oxygen and hydrogen peroxide [20], although its absence from $M$. suis makes this less likely. Genes encoding putative SodA have been reported in phytoplasmas but not other mycoplasmas, in which it is assumed that thioredoxin reductase, which is also present in both hemoplasmas, fulfils the role of superoxide scavenger. Alternatively $M$. haemofelis SodA could act as a virulence factor generating hydrogen peroxide $\left(\mathrm{H}_{2} \mathrm{O}_{2}\right)$, which damages erythrocytes. Production of reactive-oxygen species, such as $\mathrm{H}_{2} \mathrm{O}_{2}$ by virulent strains of Mycoides mycoides subsp. mycoides has been suggested as a mechanism for direct toxic effect on host cells [21]. Ribonuclease $\mathrm{R}$ has been identified as a pathogenic determinant of both Shigella flexneri and Escherichia coli, and is required for the expression of a virulent phenotype [22].
However, it has not been reported as a virulence factor in other mycoplasmas and may simply be present in $M$. haemofelis and $M$. suis for its role in messenger RNA degradation, or it may be inactive due to the $\mathrm{N}$-terminal truncation. In the absence of genes encoding the terminal organelle machinery, it is unlikely that $m g p A$ will play a role in virulence of hemoplasmas. The absence of these genes also fits with the electron-microscopy studies of $M$. haemofelis and $M$. suis that have not shown evidence of the presence of this "tip"-like structure [23-25]. Also conspicuous in their absence are the highly conserved genes encoding GroEL/ES, FtsZ and the pyruvate dehydrogenase complex, which are also absent in some ureaplasmas and are variably present in other Mollicutes. The significance of their absence is uncertain. The genome of $M$. haemofelis was however shown to contain homologs of all three of the porcine hemoplasma $M$. suis immunodominant proteins: enolase, MSG1 (GapA) and HspA1 (DnaK) $[8,26,27]$, of which MSG1 is thought to play a role in erythrocyte adhesion.

Cyclical parasitemia has been reported as a feature of M. haemofelis infection [4], whilst chronic infection is a common feature of hemoplasma infections in general [17]. We suspect that the extensive use of genome space in the metabolically restricted genomes of both hemoplasma species to encode putatively surface expressed paralogs plays a significant role in host immune system evasion, in a similar mechanism to that used by the blood-borne Rickettsial pathogen Anaplasma marginale following gene recombination between major super protein genes and their paralogs, which results in antigenic variation [28].

Previous work on the immunoproteome of $M$. haemofelis has indicated that a number of proteins of a variety of molecular weights can stimulate an immune response in the cat $[7,9]$. Of particular interest is the $25-29 \mathrm{kDa}$ region, which contains a number of immunogenic proteins identified as distinct spots on a 2D immunoblot [7], and as a variable band on one dimensional - polyacrylamide gel electrophoresis [9]. Unfortunately the majority of immunogenic spots in this region did not correspond to visible spots on gels. Further work using the more sensitive LCMSMS on defined "mass and pI regions" of the gel may be useful in indentifying these proteins, with recombinant expression of selected proteins required to further characterize their role in the immune response. Other options include using narrow range gels with increased loading, a depletion procedure to remove abundant proteins [29], or an immunoaffinity enrichment step [30]. Despite these limitations, in addition to the previously reported phosphoglycerate kinase, chaperone protein DnaK and elongation factor-Ts [7], five novel immunogenic proteins of $M$. haemofelis were identified including one of the uncharacterized protein paralogs. This, and LC-MSMS data matches to other uncharacterized protein paralogs, 
indicate that not only are hypothetical proteins from these gene duplication events expressed, but that they may also play a role in the host immune response, along with $M$. haemofelis proteins of predicted metabolic function.

In common with other Mollicutes, the genomes of $M$. haemofelis and M. suis lack many genes related to amino acid and fatty acid biosynthesis and the citric acid cycle. In the absence of genes encoding pathways for the uptake and utilization of glycerol and fructose, glucose appears to be the sole carbohydrate energy source. It is likely that this has resulted from colonization of the epierythrocytic environment that is rich in glucose, leading to such alternative energy source pathways being rendered redundant. A number of immunogenic proteins have been identified; including metabolic enzymes suspected of having multiple activities within the bacterium, and members of highly repeated families of uncharacterized hypothetical proteins suspected of playing a role in host immune system evasion. Focused MS analysis of purified hemoplasma proteins may allow further characterization of these pathogens.

\section{Additional material}

Additional file 1: Table S1: Codon usage table for Mycoplasma haemofelis str. Langford 1.

Additional file 2: Figure S1: Predicted energy metabolism pathways of Mycoplasma haemofelis str. Langford 1. Metabolic products are in black. Enzymes are in red, with direction of activity indicated by blue arrows.

Additional file 3: Table S2: Uncharacterised protein paralog families and their corresponding gene numbers.

Additional file 4: Figure S2: Representative section of the Mycoplasma haemofelis str. Langford 1 genome containing a protein paralog "island". The "Island" of protein paralog open reading frames (ORFs) is indicated by the pink arrows, with paralog family (PF) number shown. ORFs for poorly characterized proteins are in yellow, including non-repeated hypothetical proteins (HP) and HIT family protein (hit). The ORF encoding a membrane lipoprotein is in green and the ORF of metabolic enzyme nicotinate-nucleotide adenylyltransferase $(\boldsymbol{n a d D})$ is in blue. Nucleotide position is indicated by numbers and direction of read indicated by arrow head.

Additional file 5: Table S3: Mascot score and gene identity (ID) for protein spots selected for mass spectrometry analysis.

\begin{abstract}
Acknowledgements
The Roche 454 sequencing and analysis was supported by a Golden Jubilee grant from the Royal College of Veterinary Surgeons Trust Fund. The feline samples used in this study were generated from a study funded by the Wellcome Trust (Grant no. 077718). ENB was supported by a University of Bristol Postgraduate Research Scholarship and Pfizer Animal Health. Work at the University of Sydney was supported by a grant from the Worldwide Universities Network Research Mobility Programme [31], with technical assistance from Philippa Kohnke.
\end{abstract}

\section{Author details}

${ }^{1}$ School of Veterinary Sciences, University of Bristol, Langford, BS40 5DU, UK. ${ }^{2}$ Centre for Genomic Research, Institute of Integrative Biology, University of Liverpool, Liverpool, L69 7ZB, UK. 3Proteomics Facility, University of Bristol,
Bristol, BS8 1TD, UK. ${ }^{4}$ School of Chemistry, University of Bristol, Bristol, BS8 1TS, UK. ${ }^{5}$ School of Molecular Bioscience, University of Sydney, Sydney, NSW 2006, Australia. ${ }^{6}$ Institute of Infection and Global Health, University of Liverpool, Liverpool, L69 3BX, UK.

\section{Authors' contributions}

IRP and ENB prepared the M. haemofelis DNA and protein samples for analysis. MAH, ACD and ENB performed the genome analysis. ENB, KJH, CJA and $\mathrm{BC}$ performed the proteome analysis. ENB, ACD, CRH and ST co-drafted the manuscript. ST, CRH and ADR conceived the study, participated in its design and coordination. All authors read and approved the final manuscript.

\section{Competing interests}

The authors declare that they have no competing interests.

Received: 6 March 2011 Accepted: 12 July 2011 Published: 12 July 2011

\section{References}

1. Neimark H, Johansson KE, Rikihisa Y, Tully JG: Proposal to transfer some members of the genera Haemobartonella and Eperythrozoon to the genus Mycoplasma with descriptions of 'Candidatus Mycoplasma haemofelis', 'Candidatus Mycoplasma haemomuris', 'Candidatus Mycoplasma haemosuis' and 'Candidatus Mycoplasma wenyonii'. Int J Syst Evol Microbiol 2001, 51(Pt 3):891-899.

2. Barker EN, Helps CR, Peters IR, Darby AC, Radford AD, Tasker S: Complete Genome Sequence of Mycoplasma haemofelis, a hemotropic mycoplasma. J Bacteriol 2011, 193(8):2060-2061.

3. Tasker S, Caney SM, Day MJ, Dean RS, Helps CR, Knowles TG, Lait PJ, Pinches MD, Gruffydd-Jones TJ: Effect of chronic FIV infection, and efficacy of marbofloxacin treatment, on Mycoplasma haemofelis infection. Vet Microbiol 2006, 117(2-4):169-179.

4. Tasker S, Peters IR, Papasoulotis K, Cue SM, Willi B, Hofmann-Lehmann R, Knowles TG, Gruffydd-Jones TJ, Day MJ, Helps CR: Description of outcomes of experimental infection with feline haemoplasmas: haematology, Coombs' testing and blood glucose concentrations. Vet Microbio/ 2009, 139(3-4):323-332.

5. Messick JB, Santos AP, Guimaraes AM: Complete genome sequences of two hemotropic mycoplasmas - Mycoplasma haemofelis Strain Ohio2 and Mycoplasma suis Strain Illinois. J Bacteriol 2011, 193(8):2068-2069.

6. Oehlerking J, Kube M, Felder KM, Matter D, Wittenbrink MM, Schwarzenbach S, Kramer MM, Hoelzle K, Hoelzle LE: The complete genome sequence of the hemotrophic Mycoplasma suis_KI3806. J Bacteriol 2011, 193(9):2369-2370.

7. Barker EN, Helps CR, Heesom KJ, Arthur CJ, Peters IR, Hofmann-Lehmann R, Tasker S: Detection of humoral response using a recombinant heat shock protein 70 (dnaK) of Mycoplasma haemofelis in experimentally and naturally hemoplasma infected cats. Clin Vaccine Immunol 2010, 17(12):1926-1932.

8. Hoelzle LE, Hoelzle K, Harder A, Ritzmann M, Aupperle H, Schoon HA, Heinritzi K, Wittenbrink MM: First identification and functional characterization of an immunogenic protein in unculturable haemotrophic mycoplasmas (Mycoplasma suis HspA1). FEMS Immunol Med Microbiol 2007, 49(2):215-223.

9. Peters IR, Helps CR, Gruffydd-Jones TJ, Day MJ, Tasker S: Antigen specificity of the humoral immune response to Mycoplasma haemofelis infection. Clin Vaccine Immunol 2010, 17(8):1238-1243.

10. Rutherford K, Parkhill J, Crook J, Horsnell T, Rice P, Rajandream MA, Barrell B: Artemis: sequence visualization and annotation. Bioinformatics 2000, 16(10):944-945.

11. Li L, Stoeckert CJ, Roos DS: OrthoMCL: identification of ortholog groups for eukaryotic genomes. Genome Res 2003, 13(9):2178-2189.

12. Kurtz S, Phillippy A, Delcher AL, Smoot M, Shumway M, Antonescu C, Salzberg SL: Versatile and open software for comparing large genomes. Genome Biol 2004, 5(2):R12.

13. Peters IR, Helps CR, McAuliffe L, Neimark H, Lappin MR, Gruffydd-Jones TJ, Day MJ, Hoelzle K, Willi B, Meli ML, Hofmann-Lehmann R, Tasker S: RNaseP RNA gene ( $r n p B)$ phylogeny of mycoplasmas and other Mycoplasma species. J Clin Microbiol 2008, 46(5):1873-1877.

14. Cho NH, Kim HR, Lee JH, Kim SY, Kim J, Cha S, Kim SY, Darby AC, Fuxelius HH, Yin J, Kim JH, Kim J, Lee SJ, Koh YS, Jang WJ, Park KH, 
Andersson SG, Choi MS, Kim IS: The Orientia tsutsugamushi genome reveals massive proliferation of conjugative type IV secretion system and host-cell interaction genes. Proc Natl Acad Sci USA 2007, 104(19):7981-7986

15. Bai X, Zhang J, Ewing A, Miller SA, Jancso Radek A, Shevchenko DV, Tsukerman K, Walunas T, Lapidus A, Campbell JW, Hogenhout SA: Living with genome instability: the adaptation of phytoplasmas to diverse environments of their insect and plant hosts. J Bacteriol 2006, 188(10):3682-3696.

16. Minion FC, Lefkowitz EJ, Madsen ML, Cleary BJ, Swartzell SM, Mahairas GG: The genome sequence of Mycoplasma hyopneumoniae strain 232, the agent of swine mycoplasmosis. J Bacteriol 2004, 186(21):7123-7133.

17. Hoelzle LE: Haemotrophic mycoplasmas: Recent advances in Mycoplasma suis. Vet Microbiol 2008, 130:215-226.

18. Tasker S: Haemotropic mycoplasmas: what's their real significance in cats? J Feline Med Surg 2010, 12(5):369-381.

19. Berent LM, Messick JB: Physical map and genome sequencing survey of Mycoplasma haemofelis (Haemobartonella felis). Infect Immun 2003, 71(6):3657-3662

20. Gort AS, Imlay JA: Balance between endogenous superoxide stress and antioxidant defenses. J Bacteriol 1998, 180(6):1402-1410

21. Pilo P, Frey J, Vilei EM: Molecular mechanisms of pathogenicity of Mycoplasma mycoides subsp. mycoides SC. Vet J 2007, 174(3):513-521.

22. Cheng ZF, Zuo Y, Li Z, Rudd KE, Deutscher MP: The vacB gene required for virulence in Shigella flexneri and Escherichia coli encodes the exoribonuclease RNase R. J Biol Chem 1998, 273(23):14077-14080.

23. Small E, Ristic M: Morphologic features of Haemobartonella felis. Am J Vet Res 1967, 28(124):845-851.

24. Maede Y, Sonoda M: Studies on feline haemobartonellosis. III. Scanning electron microscopy of Haemobartonella felis. Nippon Juigaku Zasshi 1975, 37(2):209-211.

25. Gwaltney SM, Willard LH, Oberst RD: In situ hybridizations of Eperythrozoon suis visualized by electron microscopy. Vet Microbiol 1993 36(1-2):99-112.

26. Schreiner SA, Hoelzle K, Schwarzenbach S, Wittenbrink MM, Felder KM, Hoelzle LE: $\alpha$-Enolase - the third immunodominant antigen in Mycoplasma suis-infection. 18th Congress of The International Organisation for Mycoplasmology Chianciano Terme, Italy; 2010, 172.

27. Hoelzle LE, Hoelzle K, Helbling M, Aupperle H, Schoon HA, Ritzmann M, Heinritzi K, Felder KM, Wittenbrink MM: MSG1, a surface-localised protein of Mycoplasma suis is involved in the adhesion to erythrocytes. Microbes Infect 2007, 9(4):466-474.

28. Brayton KA, Kappmeyer LS, Herndon DR, Dark MJ, Tibbals DL, Palmer GH, McGuire TC, Knowles DP Jr: Complete genome sequencing of Anaplasma marginale reveals that the surface is skewed to two superfamilies of outer membrane proteins. Proc Natl Acad Sci USA 2005, 102(3):844-849.

29. Ringrose $J H$, van Solinge WW, Mohammed S, O'Flaherty MC, van Wijk R, Heck AJ, Slijper M: Highly efficient depletion strategy for the two most abundant erythrocyte soluble proteins improves proteome coverage dramatically. J Proteome Res 2008, 7(7):3060-3063.

30. Boschetti E, Righetti PG: The ProteoMiner in the proteomic arena: a nondepleting tool for discovering low-abundance species. J Proteomics 2008, 71(3):255-264

31. Worldwide Universities Network UK Homepage. [http://www.wun.ac.uk].

doi:10.1186/1297-9716-42-83

Cite this article as: Barker et al: Molecular characterization of the uncultivatable hemotropic bacterium Mycoplasma haemofelis. Veterinary Research 2011 42:83.

\section{Submit your next manuscript to BioMed Central and take full advantage of:}

- Convenient online submission

- Thorough peer review

- No space constraints or color figure charges

- Immediate publication on acceptance

- Inclusion in PubMed, CAS, Scopus and Google Scholar

- Research which is freely available for redistribution

Submit your manuscript at www.biomedcentral.com/submit
Biomed Central 